\title{
BMJ Open Effect of antenatal education in small classes versus standard auditorium- based lectures on use of pain relief during labour and of obstetric interventions: results from the randomised NEWBORN trial
}

\author{
Carina Sjöberg Brixval, ${ }^{1}$ Lau Caspar Thygesen, ${ }^{1}$ Solveig Forberg Axelsen, ${ }^{1}$ \\ Christian Gluud, ${ }^{2}$ Per Winkel, ${ }^{2}$ Jane Lindschou, ${ }^{2}$ Tom Weber, ${ }^{3}$ Pernille Due, ${ }^{1}$ \\ Vibeke Koushede ${ }^{1}$
}

To cite: Brixval CS, Thygesen LC, Axelsen SF, et al. Effect of antenatal education in small classes versus standard auditoriumbased lectures on use of pain relief during labour and of obstetric interventions: results from the randomised NEWBORN trial. BMJ Open 2016:6:e010761.

doi:10.1136/bmjopen-2015010761

- Prepublication history and additional material is available. To view please visit the journal (http://dx.doi.org/ 10.1136/bmjopen-2015010761).

Received 6 December 2015 Revised 18 May 2016 Accepted 19 May 2016

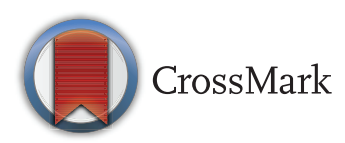

For numbered affiliations see end of article.

Correspondence to Carina Sjöberg Brixval; cabr@niph.dk

\section{ABSTRACT}

Objective: To examine the effect of an antenatal education programme in small classes versus standard auditorium-based lectures.

Design: Randomised trial using random-generated web-based 1:1 allocation.

Setting: The largest birth site in the Capital Region of Denmark, from August 2012 to May 2014.

Participants: 1766 pregnant women. Inclusion criteria $\geq 18$ years, pregnant with a single child, and able to speak and understand Danish. Women were enrolled in the trial from $10+0$ to $20+0$ weeks of gestation.

Interventions: The intervention programme consisted of three times 2.5 hours of antenatal education in small classes ( $\mathrm{n}=6-8$ women), and focused on improving information and problem-solving skills for expectant parents in order to ease birth and the transition to parenthood. The control group received standard auditorium-based lectures consisting of two times 2 hours in an auditorium with participation of 250 people.

Main outcome measures: The primary trial outcome was use of epidural analgesia. Other types of pain relief and obstetric interventions were analysed as explorative outcomes.

Results: There was no statistically significant difference in use of epidural analgesia between participants in the intervention group (30.9\%) versus the control group (29.1\%), adjusted OR 1.10 (95\% Cl 0.87 to 1.34$)$. Also, the two groups did not differ regarding other types of pain relief or obstetric interventions. Concomitant birth preparation was common in both groups and highest in the control group, but did not seem to influence our results noticeably.

Conclusions: Antenatal education in small groups versus standard auditorium-based lectures did not differ regarding use of epidural analgesia, other pain relief, or obstetric interventions.

Trial registration number: NCT01672437; Results.

\section{Strengths and limitations of this study}

- This is the largest randomised trial evaluating the effect of a structured antenatal education programme in small classes.

- We developed a programme which could be implemented in the clinical setting if proven effective and compared the programme with standard care at the largest birth site in Denmark.

- We used proper methods for reducing the risks of bias; adequate sequence generation; allocation concealment; and use of an objectively measured primary outcome, epidural analgesia, reducing the risk of bias due to non-blinding.

- Attrition was low and evenly distributed between the groups.

- A total of only $19.6 \%$ of the invited women were accepted and randomised. These women differed from the general population regarding educational level and parity. This limits the generalisability of the trial results.

\section{INTRODUCTION}

Antenatal education has the aim to provide expectant parents with strategies for dealing with pregnancy, childbirth and parenthood. ${ }^{1}$ Offers of antenatal education have undergone marked changes over time without evidence of the effect of various types of antenatal education on relevant outcomes, for example, outcomes related to birth. ${ }^{2}$ A recent systematic review concluded that insufficient evidence exist as to whether antenatal education in small classes has an effect on obstetric or psychosocial outcomes. ${ }^{3}$ 
Epidural analgesia provides effective pain relief but is associated with adverse effects on the birth process, for example, longer second stage of labour, ${ }^{45}$ and increased risk of operative birth ${ }^{4-10}$ and of caesarean section. ${ }^{5} 1011$ Use of epidural analgesia and obstetric interventions, for example, vacuum extraction and caesarean section also have economic impacts on the health system. ${ }^{12}$

Women who are anxious during labour may be at increased risk of use of epidural analgesia as pain relief due to several mechanisms; they often seek admission to the labour ward at an earlier phase of labour; ${ }^{13}$ anxiety and fear increase the risk of a longer active labour phase due to inhibited uterine contractility; ${ }^{14}$ and anxious women perceive labour as more painful ${ }^{6}$ and therefore receive more pain relief. ${ }^{15}$ Also, childbirth selfefficacy may play an important role in the risk of receiving pain relief. Women with higher levels of self-efficacy and confidence in their ability to cope with birth report lower levels of anxiety, ${ }^{16}$ perceive pain as less intensive, ${ }^{17}$ and use less epidural analgesia during labour. ${ }^{18}$ Childbirth self-efficacy may influence the timing of arrival to the labour ward, and it has been suggested that women with increased ability to cope with the early phase of labour will tend to arrive later at the labour ward. ${ }^{19} 20$

The promotion of self-efficacy beliefs during pregnancy may reduce anxiety, and this could possibly be provided through antenatal education in small classes. Antenatal education in small groups may provide an environment with the possibility for women to hear other pregnant women's experiences and for being encouraged by the other participants and the group facilitator. By these means, antenatal education in small classes may increase the woman's trust in her ability to cope with early labour, ${ }^{21}$ and thereby reduce the likelihood of early admission ${ }^{19}$ and decrease anxiety ${ }^{21}$ and experience of labour pain. ${ }^{22}$ This may in turn reduce the use of pain relief and obstetric interventions.

Antenatal education in small classes may, in addition, increase the women's knowledge uptake due to the possibility of being actively involved in the learning process. $^{23}$

Few randomised trials have examined the effect of attending antenatal education in small groups compared with other forms of education on outcomes such as the use of pain relief or obstetric interventions, ${ }^{192425}$ and among these trials conclusions are conflicting. ${ }^{3}$

Owing to the sparse evidence from randomised trials, research about the effects of antenatal education in small classes on birth-related outcomes is still needed. ${ }^{3}$ We, therefore, conducted a randomised trial to examine the effect of a general antenatal education programme in small classes versus standard education carried out as auditorium-based lectures. In this paper, we report the effect of the intervention on the primary outcome of the trial: use of epidural analgesia as well as the explorative outcomes: other types of pain relief and obstetric interventions.

\section{METHODS}

The NEWBORN trial is an individually randomised trial. The trial is registered at ClinicalTrials.gov (ID: NCT01672437), and a detailed description is published in our design article ${ }^{26}$ and in our trial protocol. ${ }^{27}$

The NEWBORN trial took place at the largest birth site in Denmark, Hvidovre Hospital, situated in the Copenhagen Capital Region. More than 6500 deliveries take place at Hvidovre Hospital each year and the catchment area comprises a diverse population regarding sociodemographic characteristics.

\section{Participants}

Women were enrolled in the trial from $10+0$ to $20+0$ weeks of gestation. Inclusion criteria were expectant women, $\geq 18$ years old at enrolment, singleton pregnancy, due to give birth at Hvidovre Hospital, and having the ability to speak and understand Danish. Exclusion criterion was not providing signed informed consent. Pregnant women were recruited from August 2012 to May 2014. The women received a written invitation to participate in the trial prior to their first visit to the midwife along with an informed consent form. Invitations were followed up by a phone call from a project employee. Initially, only primiparous women were eligible for participation, but due to slow recruitment also multiparous women were included $\sim 6$ months into the recruitment period in order to ensure adequate statistical power. ${ }^{27}$ This change in the inclusion criteria was reported to ClinicalTrials.gov.

\section{Randomisation}

Baseline data were collected using a web-based questionnaire prior to randomisation. A project employee performed individual web-based randomisation at The Copenhagen Trial Unit according to a computergenerated allocation sequence of 1:1 with varying block sizes concealed to the investigators. The allocation was stratified for parity (primiparous or multiparous) and vulnerability (yes or no as defined by their general practitioner at the first pregnancy consultation in gestation week 6-10). There were eight criteria listed for vulnerability, for example; former or current psychiatric disorder, adverse psychosocial background, or concerns about parenting skills. The general practitioner categorised the women as vulnerable if she met one or more of these criteria. For non-vulnerable women, the block sizes used for randomisation were 10 and 20, for vulnerable women the block sizes were 4 and 6 . These block sizes were used for primiparous as well as multiparous women. All the citizens in Denmark have a unique personal identification (CPR) number and the randomisation programme was set up to confirm the existence of the CPR number.

\section{Intervention group}

Women in the intervention group received an antenatal education programme-the NEWBORN programme- 
focused on parental resources important for the birth process and for parenting. In short, the programme aimed at strengthening relationships and improving information and problem-solving skills for expectant parents in order to ease birth and the transition to parenthood. The woman's partner was also invited to participate in the programme. The consulting midwife and the facilitating midwife encouraged participation of the partner. Throughout the programme, there was a focus on increasing self-efficacy in relation to the different topics touched upon, for example, childbirth selfefficacy. Also, the programme aimed at enhancing social network among the participants and highlighted the importance of partner support. The programme was designed based on the recommendations for antenatal care from the Danish Health Authority ${ }^{28}$ and developed using the Intervention Mapping approach. ${ }^{29}$ A working group consisting of midwifes, health visitors, psychologists and family therapists delivered inputs for the form and content of the programme.

A detailed description of the programme has been presented elsewhere ${ }^{26}$ and can be found as online supplementary material. Briefly, 110 groups of $\sim 6-8$ pregnant women and their partners met three times during pregnancy (gestation weeks 25, 33 and 35) for the duration of 2.5 hours per session. The sessions included among other things information and discussions about emotions and expectations related to the birth process, including information on pain relief and obstetric interventions. Accordingly, each woman was exposed to small group education for 7.5 hours during pregnancy.

The session in the 33rd week of gestation focused on pain relief and the birth process. The aim of this session was to provide the participants with information, and enhancing their existing knowledge and understanding of the normal course of labour, pain relief, and what might be expected if an obstetric intervention is necessary. For the topic on pain relief, the women were asked to discuss their thoughts and previous experiences with coping with pain and physical and mental strain, and to consider whether they could use any of these strategies during labour. Next, they discussed their thoughts and knowledge on various methods of pain relief. Plenary discussions and summing up thoughts and ideas were used so that participants could learn from and be inspired by one another. These methods were used to enhance the women's childbirth self-efficacy.

Also, the participants had access to a patient network website specifically developed for the NEWBORN trial to gain further information, communicate with other participants in the trial, and consult online with a midwife and a health visitor. At each session, the participants were encouraged by the instructors to use the website.

A total of 25 midwives with varying professional seniority and teaching experience facilitated the 110 classes. They enrolled for teaching themselves and were not specifically selected by the trial investigators. The instructors followed a detailed teaching manual developed for the trial. ${ }^{30}$

In the Copenhagen area, different kinds of birth and parent education offers are provided by private stakeholders. These offers include, for example, mindfulness training, physical exercise training and mental preparation for delivery. Participants in the intervention group were permitted to make use of concomitant birth and parent education.

\section{Control group}

Women in the control group received the standard education offered from Hvidovre Hospital consisting of two antenatal lectures of 2 hours, each on birth and breast feeding in an auditorium with participation of up to 250 people. Accordingly, each woman was exposed to large group education for 4 hours. The content of the lecture on birth included information on, for example, what to do at home when labour had begun; information on the location of labour ward at the hospital; phases of the labour and information on different types of pain relief. The form was passive information giving from a midwife to the participants in the lectures.

Midwives conducting the lectures volunteered for the teaching. To avoid contamination of conditions, midwives facilitating the group-based experimental programme were not allowed to teach the antenatal lectures in the control group.

In addition to participants in the intervention group, participants in the control group were permitted to make use of concomitant birth and parent education.

\section{Blinding}

It was not possible to blind participants or service providers. The outcome assessors; midwives, and physicians at the labour ward were not informed about the women's participation in the trial. Data were blinded by a data manager and the investigators were therefore blinded to participants' intervention category during data assessment and analyses. Participants' intervention category was not revealed to the investigators until the Steering Committee of the trial had drawn two conclusions about intervention effects on outcomes under code..$^{31}$

\section{Variables}

The primary outcome of the trial was the use of epidural analgesia during labour. The use of other types of pain relief and obstetric interventions was examined as explorative outcomes.

Data on the use of pain relief, obstetric interventions, and other variables related to the birth was assessed from the hospital-based register at Hvidovre Hospital, the Obstetric Database. All births performed at Hvidovre Hospital and two other birth sites in the Capital Region are included in this database and entries are made by CPR number. No information about birth was collected specifically for the NEWBORN trial. In a validation 
study, we found that the validity of information on epidural analgesia and selected obstetric interventions was high in the obstetric database when using the medical records as the gold standard. ${ }^{33}$ The positive predictive values for epidural analgesia, vacuum extraction and emergency caesarean section were 1.00 , and for elective caesarean section the positive predictive value was $0.96 .^{33}$

The following variables were used for examination of baseline differences: Educational level was measured by the question: 'What is your highest completed education'? The educational level was dichotomised into $\leq$ medium tertiary education versus higher tertiary education. Body mass index $\left(\mathrm{kg} / \mathrm{m}^{2}\right)$ was calculated using the information on prepregnancy weight and height reported by the woman at the first pregnancy consultation at the general practitioner. Living with child's father was self-reported by ticking the response category 'Living with the child's father' in the question: 'Which grown-ups do you live with'? Planned pregnancy was selfreported by the question: 'Is this pregnancy planned, partly planned or not planned' and dichotomised into: planned (yes or partly) versus not planned. Self-rated physical and mental health was measured by the items: 'How would you describe your physical/mental health status altogether'? Response categories: 'Excellent, very good, good, poor, very poor'. Self-rated physical/mental health was dichotomised into excellent/very good versus good, poor, very poor. Feeling of stress was measured by the item: 'Do you feel stressed'? Response categories: 'no; yes, a little; yes, moderately; yes, a lot'. Stress was dichotomised into no versus yes, a little; yes, moderately; yes, a lot. Antenatal depressive symptomatology was measured by the Edinburgh Postnatal Depression Scale ${ }^{34}$ posed in the baseline questionnaire. A score of 13 or more were categorised as antenatal depressive symptomatology. Perceived stress was measured by the Perceived Stress Scale. ${ }^{35}$

In order to give an indication of the quality of the delivery of the programme, we assessed adherence to the programme by tablet-based questionnaires. After each session, the participants were asked whether they had been through the topics of the day. For example, after session 2, the participants were asked: 'Have you heard about "coping with pain and pain relief" today'. Participants could answer 'yes', 'no', or 'don't know'.

Data on use of concomitant birth and parent education were collected by questionnaires at gestation week 37 and 9 weeks after birth. We examined the prevalence of antenatal depressive symptomatology among participants as a potential adverse outcome. Participants in the intervention group could potentially have experienced more antenatal depressive symptoms, for example, due to a raised awareness on couple communication and potential relationship problems through the sessions. Antenatal depressive symptomatology was measured by the Edinburg Postnatal Depression Scale ${ }^{34}$ collected by questionnaire in gestation week 37. Although initially developed for measuring depressive symptoms in the postnatal period, the scale has been validated for use during pregnancy as well. ${ }^{36}$ Women with a score of 13 or more were categorised with antenatal depressive symptomatology as recommended in a former Swedish study. ${ }^{36}$

\section{Sample size}

The sample size calculation was based on the primary outcome of the trial, use of epidural analgesia. Previous data from trials ${ }^{19}$ and hospital registers ${ }^{37}$ indicate that the proportion of women who use epidural analgesia is between $23 \%$ and $41 \%$. We assumed that $31 \%$ in the control group would receive epidural analgesia and that this proportion could be reduced to $25 \%$ in the intervention group (a relative risk reduction of 19\%). Our original sample size calculation was based on a power of 0.90 and a significance level of 0.05 requiring randomisation of 2350 women to detect significant intervention effects. However, due to slow recruitment power was reduced to 0.80 requiring randomisation of 1756 women. This sample size adjustment was carried out after inclusion of 1050 participants without inspection of the data. ${ }^{27} 38$

\section{Statistical analysis}

Data were planned to be analysed according to the intention-to-treat principle and following the recommendations of the CONSORT statement. ${ }^{39} 40$

\section{Main analyses}

Differences in frequency of use of epidural analgesia, other types of pain relief, and obstetric interventions between the two groups were tested in logistic regression models adjusted for the protocol-specified stratification variables; parity and vulnerability. ORs and $95 \%$ CIs, as well as relative risk (RR) estimates with 95\% CI were calculated. Difference in the proportion of the adverse outcome antenatal depressive symptomatology between the groups was tested by $\chi^{2}$ test.

\section{Handling of missing data}

We tested whether missing values of the primary outcome, epidural analgesia, were missing completely at random (MCAR) by Little's test. ${ }^{41}$ Also, 'worst case' and a 'best case' scenario analyses of the potential impact of missing values were conducted. In the worst-case scenario, missing values of epidural analgesia in the intervention group were imputed by a 'yes' and missing values of the control group were imputed by a 'no'. In the bestcase scenario, missing values of epidural analgesia in the intervention group were imputed by a 'no' and missing values of the control group were imputed by a 'yes'.

We selected participants with full report on the primary outcome for the modified intention-to-treat analysis (see results). 
Sensitivity and per-protocol analyses

We conducted a post hoc analysis with the aim of examining the impact of concomitant birth and parent preparation on the primary outcome. From the modified intention-to-treat cohort, we excluded the participants who made use of concomitant birth and parent education in both intervention groups.

The compliance with the randomised interventions was not $100 \%$. We therefore planned per-protocol analyses in our trial protocol. Definition of per-protocol conditions were carried out prior to data analysis. The results from the per-protocol analyses are interpreted as explorative. We compared the use of epidural analgesia between the two intervention groups in per-protocol populations defined as follows:

1. Participants in the intervention group who participated in all three sessions before birth and used the website at least once versus all participants in the control group were selected from the modified intention-to-treat cohort.

2. Participants in the intervention group who participated in all three sessions before birth and used the website at least once versus participants in the control group who participated in both antenatal lectures were selected from the modified intention-to-treat cohort.

All statistical analyses were performed using SAS V. 9.3, SAS Institute Inc. The level of significance was set to 0.05 .

\section{RESULTS}

\section{Participant flow and baseline data}

During the recruitment period, 8997 women were invited to participate in the NEWBORN trial. Of these, 1766 women $(19.6 \%)$ accepted participation and were randomised-883 women to the intervention group versus 883 to the control group. At baseline, the characteristics among the intervention and control groups seem well balanced (table 1).

The attrition was similar in the two groups (figure 1). Little's test for MCAR was insignificant $(p=0.64)$. Therefore, no imputation of missing values was performed. The modified intention-to-treat analysis therefore included 1711 participants (858 in the intervention group vs 853 in the control group).

\section{Effect of the experimental intervention}

We found no effect of the NEWBORN intervention. Among women in the intervention group, $30.5 \%$ received epidural analgesia compared with $29.1 \%$ in the control group (adjusted $\mathrm{OR}=1.10$ (0.87 to 1.34 ), $\mathrm{p}=0.41$ ). None of the exploratory outcomes differed statistically between the two groups (table 2). We found no adverse effects of attending the experimental group on antenatal depressive symptomatology. The proportion of participants categorised as having antenatal depressive symptomatology at gestation week 37 was $5.6 \%$ in the intervention group and $6.8 \%$ in the control group $(\mathrm{p}=0.34)$.
Table 1 Baseline characteristics of women enrolled in the NEWBORN trial $(n=1766)$

\begin{tabular}{|c|c|c|}
\hline & $\begin{array}{l}\text { Experimental } \\
(\mathrm{n}=883)\end{array}$ & $\begin{array}{l}\text { Control } \\
(n=883)\end{array}$ \\
\hline $\begin{array}{l}\text { Age at birth in years (mean } \\
(\mathrm{SD}))^{\star}\end{array}$ & $30.7(4.1)$ & $30.8(4.1)$ \\
\hline Nulliparous, n (\%) & $787(89.1)$ & 785 (88.9) \\
\hline Vulnerable women, n (\%) & $42(4.8)$ & $42(4.8)$ \\
\hline $\begin{array}{l}\text { Educational level (higher } \\
\text { tertiary education), n (\%) }\end{array}$ & 659 (75.6) & $663(76.5)$ \\
\hline $\begin{array}{l}\text { Body mass index } \mathrm{kg} / \mathrm{m}^{2} \\
(\text { mean }(\mathrm{SD}))^{*}\end{array}$ & $23.4(4.0)$ & $23.3(4.1)$ \\
\hline $\begin{array}{l}\text { Living with child's father } \\
\text { (yes), } \mathrm{n}(\%)\end{array}$ & $828(93.8)$ & $848(96.0)$ \\
\hline $\begin{array}{l}\text { Planned pregnancy (yes/ } \\
\text { partly), } \mathrm{n}(\%)\end{array}$ & 801 (90.9) & $808(91.5)$ \\
\hline $\begin{array}{l}\text { Self-rated physical health } \\
\text { status (excellent/very } \\
\text { good), } \mathrm{n}(\%)\end{array}$ & $605(68.6)$ & $628(71.2)$ \\
\hline $\begin{array}{l}\text { Self-rated mental health } \\
\text { status (excellent/very } \\
\text { good), } n(\%)\end{array}$ & $635(72.0)$ & 669 (75.9) \\
\hline Not feeling stressed, n (\%) & 425 (48.2) & $433(49.2)$ \\
\hline $\begin{array}{l}\text { Edinburgh Postnatal } \\
\text { Depression Scale score of } \\
13 \text { or more, } n(\%)\end{array}$ & $42(4.8)$ & $28(3.2)$ \\
\hline $\begin{array}{l}\text { Perceived Stress Scale } \\
\text { score (mean (SD)) }\end{array}$ & $12.5(5.2)$ & $12.2(5.2)$ \\
\hline
\end{tabular}

We conducted 'worst-case' and 'best-case' scenario analyses to assess the potential impact of missing values. Results from the best-case scenario showed no difference between intervention group and control group on the use of epidural analgesia (adjusted $\mathrm{OR}=0.93$ ( 0.76 to 1.14), $\mathrm{p}=0.49)$. In the worst-case scenario, the results indicated a negative impact of the intervention (adjusted $\mathrm{OR}=1.25$ (1.02 to 1.54$), \mathrm{p}=0.03$ ) (see online supplementary table $\mathrm{S} 1)$.

\section{Adherence to the programme in session 2}

To give an indication of the quality of delivery of the programme, we assessed the facilitator's adherence to the programme content in session 2. Adherence was reported high by the participants. More than $97 \%$ of the participants reported to have heard about the topics: 'expectations in relation to birth', 'what to do at home in the early phase of labour', 'the normal course of labour, pain relief and coping strategies', and 'partner support during labour'. A total of $88 \%$ of the participants reported having been through the topic "when there is a need to intervene in labour'.

\section{Use of birth and parent education offers}

Use of birth and parent education offers was unequally distributed among intervention groups (see online supplementary table S2). There were a considerably higher 


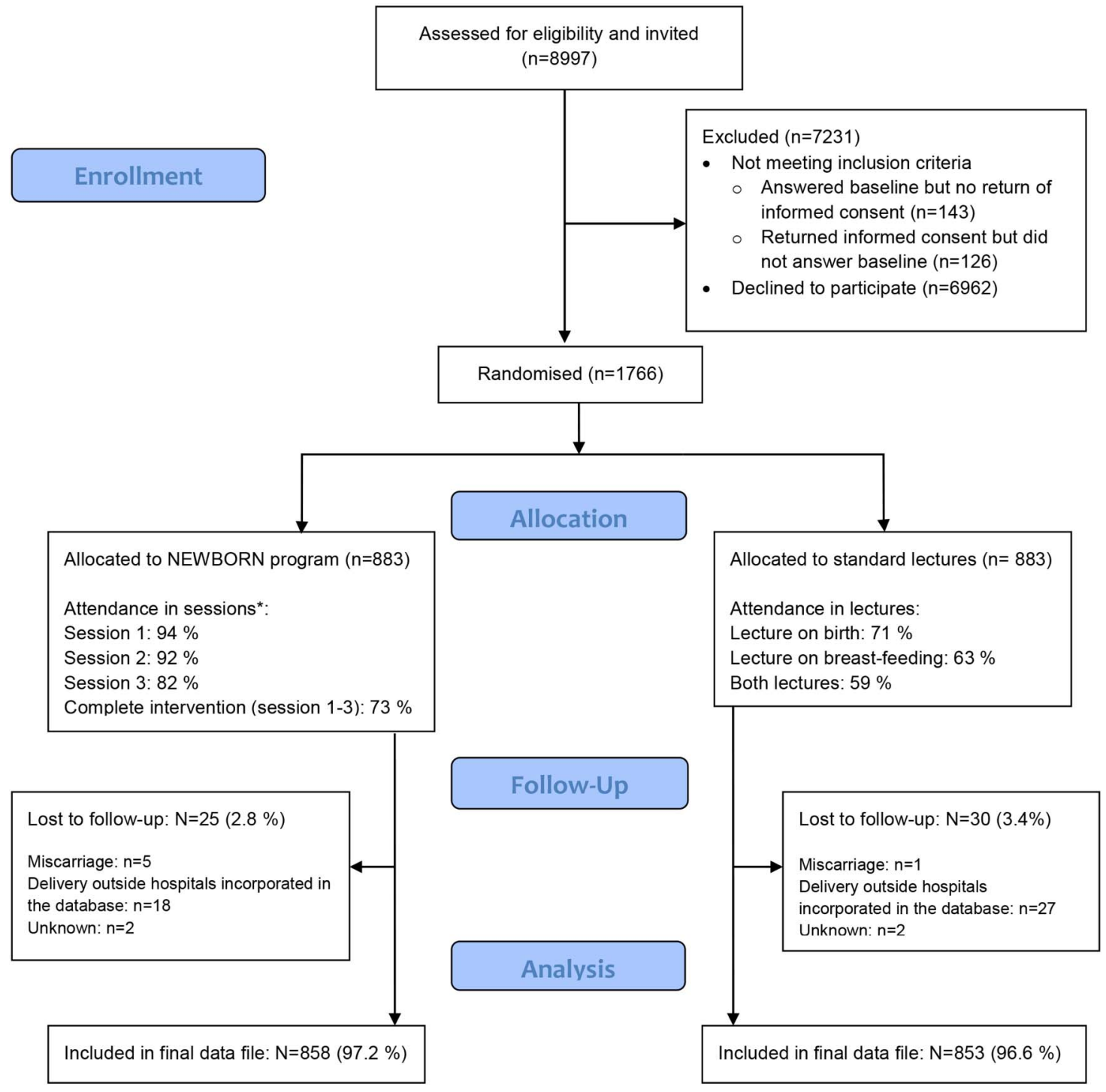

* Measurement of attendance is obtained by participant's responses to questionnaires. Proportions of attendance are based on the following response proportions: complete intervention: $72 \%$, session 1: $86 \%$, session 2: $80 \%$, session 3: $78 \%$.

Figure 1 Flow diagram of recruitment, randomisation and participation in the NEWBORN trial.

proportion of participants in the control group (38.7\%) who attended other types of birth and parent preparation offers than among participants in the intervention group $(25.0 \%)$. Also, there were more participants who did not attend any birth and parent education offers in the control group (11\%) than among participants in the intervention group $(2.5 \%)$.

\section{Additional analyses}

We performed an additional sensitivity analysis examining the effect of the intervention on the use of epidural analgesia excluding women who made use of concomitant preparation education. This reduced the sample from 1711 women to 1052 women. Results were similar to the results from the modified intention-to-treat analysis, that is, there was no effect of the intervention (table 3). In the per-protocol analyses where we examined the effect of the intervention among participants adhering to the intervention, the sample was reduced with $\sim 25 \%$. Also, results from these analyses were consistent with the results from the modified intention-to-treat analysis (table 3).

\section{DISCUSSION}

The results from this randomised trial showed that the experimental education consisting of small classes for 7.5 hours versus control education with large group lectures for 4 hours gave no difference in the use of epidural analgesia, other types of pain relief during labour or obstetric interventions. Use of private birth and parent preparation offers were considerably higher 







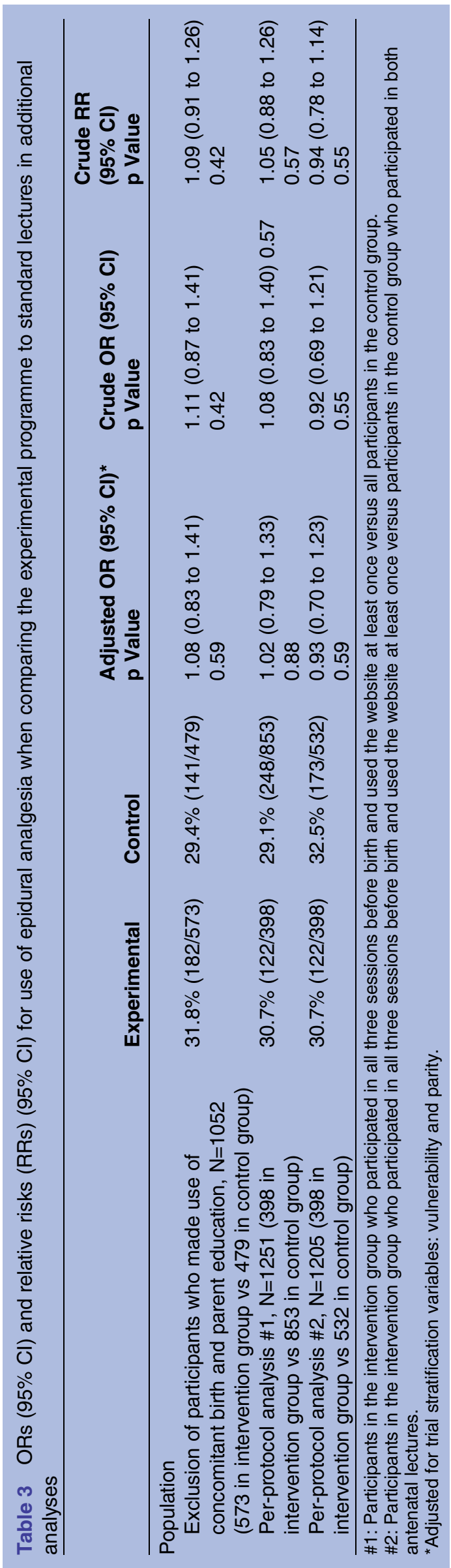

among participants in the control group compared with participants in the intervention group, but also no participation in birth and parent preparation was more frequent in the control group than among participants in the intervention group. We examined the impact of the concomitant education by excluding women that participated in other education and found that this exclusion did not alter our results noticeably.

We hypothesised that the NEWBORN programme would increase childbirth self-efficacy and by this reduce the use of pain relief. We have examined the effect of the programme on the intermediate trial outcome; childbirth self-efficacy measured by three single items developed for the NEWBORN trial. In the intervention group, $4.1 \%$ of the women had low confidence in their own ability to cope with early phase of labour before going to the labour ward compared with $8.0 \%$ in the control group. Fewer women in the intervention group $(5.0 \%)$ felt low confidence in their own ability to handle the birth process no matter how it turns out compared with the control group $(7.4 \%) .{ }^{42}$ Hence, these results suggest that although the intervention had no effect on the epidural analgesia, the programme may have the potential to enhance the women's childbirth selfefficacy. Former studies have found that women with higher levels of self-efficacy perceive pain as less intensive $^{17}$ and use less epidural analgesia during labour ${ }^{18}$ compared with women with lower levels of self-efficacy. The potential associations between childbirth selfefficacy and experience of pain and use of epidural analgesia have not been investigated in the present study.

Only three randomised trials have examined the effect of attending antenatal education in small groups compared with other forms of education on outcomes, such as the use of pain relief or obstetric interventions. ${ }^{19} 2425$ Two of these trials were performed among women screened positive for fear of childbirth limiting generalisation of results to the general population. ${ }^{24}{ }^{25}$ One former Danish trial ${ }^{19}$ examined the effect of antenatal education classes versus no education among 1193 primiparous women recruited among a diverse population group not limited to a high-risk population. This trial by Maimburg et $a l^{19}$ is comparable to our NEWBORN trial regarding the included population, but they compared small classes versus no intervention. Maimburg et $a l^{19}$ reported a statistically significant reduced use of epidural analgesia in their experimental group, but not of other types of pain relief and obstetric interventions. The two trials differ regarding the control intervention and we included primiparous and multiparous women, whereas Maimburg et al only included primiparous. Furthermore, we used 25 voluntary midwives with varying teaching experience, whereas in the trial by Maimburg et al classes were taught by four selected midwives. Also, the midwives in the Maimburg trial may have gained more teaching experience during the trial period compared with the midwives in the NEWBORN trial, as some of the midwives in our trial taught only a few classes. 


\section{Strengths and limitations}

This randomised trial is to our knowledge the largest trial assessing the effect of antenatal education in small classes versus auditorium-based lectures. The intervention was developed using a systematic framework for health promotion programme planners. ${ }^{29}$ This systematic framework aids effective decision-making at each step in intervention planning, implementation and evaluation. We focused on conducting a trial using standard care as control condition and tested a birth and parent preparation programme that would be feasible to implement in an everyday clinical practice setting if proven effective. We chose a control condition that is relevant to public health; standard care instead of a different antenatal intervention, and the study population was recruited among a diverse population group and not limited to a high-risk population. The attrition was low $(3 \%)$ and distributed evenly between the intervention and control groups.

It was not possible to blind participants or educators which may introduce bias. However, using an objective primary outcome, such as epidural analgesia, reduces the risk of bias due to lack of blinding. ${ }^{43} 44$ The outcome assessors, midwives at the labour ward, were not informed about the women's participation in the trial but it cannot be ruled out that the women informed the midwife about their intervention status. However, we consider it unlikely that the decision to provide pain relief or perform obstetric interventions rely on the intervention status as such decisions are made by the midwives and physicians at the labour ward.

Initially, only primiparous women were eligible for inclusion in the trial. During the recruitment period, we allowed for inclusion of multiparous women. This was carried out for practical reasons to ensure adequate power in the analyses. Although this change was reported to the clinical trial register, the posterior inclusion of multiparous women must be considered a limitation.

Of the 8997 pregnant women invited to participate in the trial, only $19.6 \%$ were accepted and were randomised. Although we aimed to recruit a diverse population group to the NEWBORN trial, the participants were predominantly primiparous women and women with a higher education level compared to the general population of Copenhagen women in the same age group. ${ }^{45}$ The high proportion of women with a university education in the trial population may imply that the women included in the trial find this teaching form more appealing than the general population. Moreover, the proportion receiving pain relief and obstetric interventions (except elective caesarean section) were higher among the trial population than among the total population of women giving birth at Hvidovre Hospital. ${ }^{46}$ These discrepancies between the trial population and background population characteristics may limit the generalisability of the trial results, and the intervention might have different effects among multiparous or women of a lower educational level. It may be beneficial to conduct research focusing on the effect of the programme among subgroups, for example, women with lower educational level or vulnerable women. Also, further analyses taking adherence to the programme into consideration would contribute with more thorough knowledge of the impact of the programme. These issues need to be investigated before recommendation of implementation of the programme in clinical practice can be validly expressed.

\section{CONCLUSIONS}

The results from the NEWBORN trial showed no difference in use of epidural analgesia, other types of pain relief during labour, or obstetric interventions between women randomised to antenatal education in small classes versus standard lectures. The effects of the intervention on the secondary outcomes of the NEWBORN trial: perceived stress, parenting stress, and parenting alliance will be reported in later articles.

\section{Author affiliations}

${ }^{1}$ National Institute of Public Health, University of Southern Denmark, Copenhagen, Denmark

${ }^{2}$ Copenhagen Trial Unit, Centre for Clinical Intervention Research, Rigshospitalet, Copenhagen University Hospital, Copenhagen, Denmark ${ }^{3}$ Department of Obstetrics and Gynaecology, Hvidovre Hospital, Copenhagen University Hospital, Hvidovre, Denmark

Acknowledgements The authors would like to acknowledge all participants in the NEWBORN trial as well as the NEWBORN Trial Group ${ }^{26}$ involved in the trial. They thank the Danish Cancer Society for funding of the trial. Moreover, they also thank Janus Engstrøm, Copenhagen Trial Unit, for development of the randomisation system and provision of allocation sequence; Steen Christian Rasmussen, Hvidovre Hospital, for extraction of data from the Obstetric Database and Bjarne Laursen, National Institute of Public Health, for valuable help with data management.

Contributors CSB, SFA, PD and VK developed the study design in collaboration with TW, JL and CG. PW wrote the statistical analysis plan. CSB, SFA and VK collected the data. CSB and LCT conducted the statistical analyses. CSB drafted the manuscript. All authors have been involved with interpretation of results, critical revision of the manuscript and final approval of the submitted manuscript.

Funding This work was supported by the Danish Cancer Society. The funders have not been involved in trial design, data collection and analysis, or decision to publish this manuscript.

Competing interests None declared.

Ethics approval The NEWBORN trial has been assessed by and registered in the Capital Region's ethics committee 23 March 2012 (CVR/SE number 30113713) and registered and listed in the Danish Data Protection Agency (reference number 2011-54-1289).

Provenance and peer review Not commissioned; externally peer reviewed.

Data sharing statement The authors commit to making the relevant anonymised patient-level data available on reasonable request. Please contact the corresponding author.

Open Access This is an Open Access article distributed in accordance with the Creative Commons Attribution Non Commercial (CC BY-NC 4.0) license, which permits others to distribute, remix, adapt, build upon this work noncommercially, and license their derivative works on different terms, provided the original work is properly cited and the use is non-commercial. See: http:// creativecommons.org/licenses/by-nc/4.0/ 


\section{REFERENCES}

1. Ahlden I, Ahlehagen S, Dahlgren LO, et al. Parents' expectations about participating in antenatal parenthood education classes. J Perinat Educ 2012;21:11-17.

2. Gagnon AJ, Sandall J. Individual or group antenatal education for childbirth or parenthood, or both. Cochrane Database Syst Rev 2007;(3):CD002869.

3. Brixval CS, Axelsen SF, Lauemøller SG, et al. The effect of antenatal education in small classes on obstetric and psycho-social outcomes-a systematic review. Syst Rev 2015;4:1-9.

4. Anim-Somuah M, Smyth RM, Jones L. Epidural versus non-epidural or no analgesia in labour. Cochrane Database Syst Rev 2011;(12): CD000331.

5. Zimmer EZ, Jakobi P, Itskovitz-Eldor J, et al. Adverse effects of epidural analgesia in labor. Eur J Obstet Gynecol Reprod Biol 2000;89:153-7.

6. Jones $\mathrm{L}$, Othman $\mathrm{M}$, Dowswell $\mathrm{T}$, et al. Pain management for women in labour: an overview of systematic reviews. Cochrane Database Syst Rev 2012;3:CD009234.

7. Poignant M, Hjelmstedt A, Ekeus C. Indications for operative delivery between 1999-2010 and induction of labor and epidural analgesia on the risk of operative delivery - a population based Swedish register study. Sex Reprod Healthc 2012;3:129-34.

8. Nguyen US, Rothman KJ, Demissie S, et al. Epidural analgesia and risks of cesarean and operative vaginal deliveries in nulliparous and multiparous women. Matern Child Health $\mathrm{J}$ 2010;14:705-12.

9. Kjaergaard $\mathrm{H}$, Olsen $\mathrm{J}$, Ottesen $\mathrm{B}$, et al. Obstetric risk indicators for labour dystocia in nulliparous women: a multi-centre cohort study. BMC Pregnancy Childbirth 2008;8:45.

10. Eriksen LM, Nohr EA, Kjaergaard H. Mode of delivery after epidural analgesia in a cohort of low-risk nulliparas. Birth 2011;38:317-26

11. Bannister-Tyrrell M, Ford JB, Morris JM, et al. Epidural analgesia in labour and risk of caesarean delivery. Paediatr Perinat Epidemiol 2014;28:400-11.

12. Tracy SK, Tracy MB. Costing the cascade: estimating the cost of increased obstetric intervention in childbirth using population data. BJOG 2003;110:717-24.

13. Carlsson IM, Hallberg LR, Odberg Pettersson K. Swedish women's experiences of seeking care and being admitted during the latent phase of labour: a grounded theory study. Midwifery 2009;25:172-80.

14. Lederman RP, Lederman $\mathrm{E}$, Work BA Jr, et al. The relationship of maternal anxiety, plasma catecholamines, and plasma cortisol to progress in labor. Am J Obstet Gynecol 1978;132:495-500.

15. Alehagen S, Wijma K, Wijma B. Fear during labor. Acta Obstet Gynecol Scand 2001;80:315-20.

16. Lowe NK. Self-efficacy for labor and childbirth fears in nulliparous pregnant women. J Psychosom Obstet Gynaecol 2000;21:219-24.

17. Lowe NK. The pain and discomfort of labor and birth. J Obstet Gynecol Neonatal Nurs 1996;25:82-92.

18. Carlsson IM, Ziegert K, Nissen E. The relationship between childbirth self-efficacy and aspects of well-being, birth interventions and birth outcomes. Midwifery 2015;31:1000-7.

19. Maimburg RD, Vaeth M, Durr J, et al. Randomised trial of structured antenatal training sessions to improve the birth process. BJOG 2010;117:921-8.

20. Beebe KR, Lee KA, Carrieri-Kohlman V, et al. The effects of childbirth self-efficacy and anxiety during pregnancy on prehospitalization labor. J Obstet Gynecol Neonatal Nurs 2007;36:410-18.

21. Taheri Z, Mazaheri MA, Khorsandi M, et al. Effect of educational intervention on self-efficacy for choosing delivery method among pregnant women in 2013. Int J Prev Med 2014;5:1247-54

22. Ip WY, Tang CS, Goggins WB. An educational intervention to improve women's ability to cope with childbirth. J Clin Nurs 2009;18:2125-35.

23. Illeris K. Contemporary theories of learning: learning theorists...in their own words. Routledge, 2009.
24. Werner A, Uldbjerg N, Zachariae R, et al. Self-hypnosis for coping with labour pain: a randomised controlled trial. BJOG 2013;120:346-53.

25. Rouhe H, Salmela-Aro K, Toivanen $\mathrm{R}$, et al. Obstetric outcome after intervention for severe fear of childbirth in nulliparous womenrandomised trial. BJOG 2013;120:75-84.

26. Koushede V, Brixval CS, Axelsen SF, et al. Group-based antenatal birth and parent preparation for improving birth outcomes and parenting resources: study protocol for a randomised trial. Sex Reprod Healthc 2013;4:121-6.

27. Koushede V. Project Newborn-Preparation for Birth and Parenthood 2014. http://www.interventionsforskning.dk/userfiles/files/nyfdt/ projektprotokol-version-23_2.pdf (accessed 7 Aug 2015).

28. Danish Health Authority. Recommendations for Antenatal Care (in Danish). Copenhagen, 2009. http://sundhedsstyrelsen.dk/publ/ Publ2009/CFF/gravide/Svangreomsorgen.pdf (accessed 9 Mar 2015).

29. Bartholomew LK, Parcel GS, Kok G, et al. Planning health promotion programs: an intervention mapping approach. John Wiley \& Sons, 2011

30. Axelsen SF, Brixval CS, Koushede V. Course material for teachers: NEWBORN-preparation for birth and parenthood. 2012.

31. Gotzsche PC. Blinding during data analysis and writing of manuscripts. Control Clin Trials 1996;17:285-90; discussion 90-3.

32. Jarvinen TL, Sihvonen R, Bhandari M, et al. Blinded interpretation of study results can feasibly and effectively diminish interpretation bias. $J$ Clin Epidemiol 2014;67:769-72.

33. Brixval CS, Thygesen LC, Johansen NR, et al. Validity of a hospital-based obstetric register using medical records as reference. Clin Epidemiol 2015;7:509-15.

34. Cox JL, Holden JM, Sagovsky R. Detection of postnatal depression. Development of the 10-item Edinburgh Postnatal Depression Scale. Br J Psychiatry 1987;150:782-6.

35. Cohen S, Kamarck T, Mermelstein R. A global measure of perceived stress. J Health Soc Behav 1983;24:385-96.

36. Rubertsson C, Borjesson K, Berglund A, et al. The Swedish validation of Edinburgh Postnatal Depression Scale (EPDS) during pregnancy. Nord J Psychiatry 2011;65:414-18.

37. Hvidovre Hospital. Births in numbers 2012 (in Danish). https://www. hvidovrehospital.dk/afdelinger-og-klinikker/foedeomraadet/omafdelingen/Sider/Foedsler-i-tal.aspx (accessed 9 June 2013).

38. ClinicalTrials.gov. NewPreBP: Project Newborn-Preparation for Birth and Parenthood. https://clinicaltrials.gov/ct2/results? term=NCT01672437 Last update: December 2015 (accessed 3 Dec 2015).

39. Moher D, Hopewell S, Schulz KF, et al. CONSORT 2010 Explanation and Elaboration: updated guidelines for reporting parallel group randomised trials. J Clin Epidemiol 2010;63: e1-37.

40. Boutron I, Moher D, Altman DG, et al. Extending the CONSORT statement to randomized trials of nonpharmacologic treatment: explanation and elaboration. Ann Intern Med 2008;148:295-309.

41. Little RJA. A test of missing completely at random for multivariate data with missing values. J Am Stat Assoc 1988;83:1198-202.

42. Brixval CS, Axelsen SF, Thygesen LC, et al. Antenatal education in small classes may increase childbirth self-efficacy: results from a Danish randomised trial. Sex Reprod Healthc 2016, in press. doi:10.1016/j.srhc.2016.03.003

43. Wood L, Egger M, Gluud LL, et al. Empirical evidence of bias in treatment effect estimates in controlled trials with different interventions and outcomes: meta-epidemiological study. BMJ 2008;336:601-5.

44. Savovic J, Jones HE, Altman DG, et al. Influence of reported study design characteristics on intervention effect estimates from randomized, controlled trials. Ann Intern Med 2012;157:429-38.

45. Statistics Denmark. Population highest completed education 2014. http://www.statistikbanken.dk/statbank5a/default.asp?w=1422 (accessed 16 Mar 2015).

46. Hvidovre Hospital. Births in numbers 2014 (in Danish). https://www. hvidovrehospital.dk/afdelinger-og-klinikker/foedeomraadet/ om-afdelingen/Sider/Foedsler-i-tal.aspx (accessed 7 Aug 2015). 